\title{
A cogniçãa no processo de design
}

The cognition in the design process

Tiago Barros Pontes e Silva

design, cognição, heurísticas, método
O objetivo do presente relato é o de apresentar uma abordagem de compreensão do processo de design a partir do referencial da Psicologia Cognitiva, especialmente ancorados em modelos de arquitetura cognitiva e em abordagens de resolução de problemas, visando sugerir um processo de metacognição para os designers. É apresentada uma abordagem de design enquanto processo de resolução de problemas, incluindo heurísticas comuns em tais processos, as suas naturezas de análise e síntese e contribuições da Psicologia Cognitiva em processos de metacognição, criatividade e avaliação.

The purpose of this document is to present an approach of understanding of the design process from the framework of cognitive psychology. It is anchored in models of cognitive architecture and problem-solving approaches in order to suggest a metacognition practice for the designers. Is presented an approach of design as a process of problem solving, including common heuristics, their analysis and synthesis nature and contributions from Cognitive Psychology in metacognition processes, creativity and evaluation.

\section{Introdução}

Atualmente, são muito frequentes as discussões acerca dos processos de design e suas aplicações em outras áreas de conhecimento. A perda da tangibilidade dos objetos de projeto, a diversidade e capilaridade das tecnologias atuais em movimentos de software e hardware livres, assim como a demanda por celeridade em processos de gestão e de concepção de sistemas informacionais ilustram a necessidade de compreensão de contextos complexos com relacionamento dinâmico de seus interagentes, influenciando a demanda por um processo sistemático de pensamento criativo.

Nesse contexto, dentro do campo projetual atribuído à atividade de design, se faz pertinente o aprimoramento dos processos de planejamento e proposição de soluções formais, estruturais ou relacionais aos produtos, ambientes, sistemas ou serviços designados, com intuito de adequar e otimizar seus métodos de trabalho ao diversificado contexto político, social, econômico e ambiental em que vivemos. 
Assim, propõe-se o estudo dos processos decisórios relacionados à busca de soluções criativas em contextos de resolução de problemas, circunstâncias muito frequentes em situações de projeto. Nesse sentido, o objetivo do presente relato é o de apresentar uma abordagem de compreensão do processo de design a partir do referencial da Psicologia Cognitiva, especialmente ancorados em modelos de arquitetura cognitiva e em abordagens de resolução de problemas, visando a possibilidade de um processo de metacognição por parte dos designers na concepção e avaliação dos projetos.

\section{Design enquanto processo de Resolução de Problemas}

Para diversos autores, a abordagem do design é frequentemente associada ao contexto de Resolução de Problemas, ou seja, o projeto é orientado a uma questão externa a ser transformada, de cunho social, comercial ou ambiental (Bonsiepe, 1984; Munari, 1998; Maldonado, 1999; Papenek, 2000; Löbach, 2001; Vassão, 2010). Ao compreender a ação de design como um artifício para a solução de um problema, busca-se a Teoria Espacial de Newell e Simon (1972, em Sterberg, 2000) para elucidar melhor a fundamentação do processo de design.

Em sua teoria, os autores afirmam que a resolução de um problema é antecedida por um processo de compreensão e busca na construção do Espaço do Problema. Esse espaço é composto por diferentes estados, que separam a situação atual do contexto da situação intencionada. Assim, os problemas são compostos pelos seguintes elementos: (a) Estado Inicial, (b) Estado Final (objetivos), (c) Estados Intermediários, (d) os Obstáculos presentes no caminho e (e) os Operadores, entendidos como alternativas possíveis para a desobstrução dos obstáculos.

O Espaço do Problema é circunscrito por operações que nem sempre obedecem a um percurso pré-determinado, na medida em que permitem atalhos, para finalmente configurar o seu Estado Final (EF). Nesse processo, o Estado Inicial (EI) é modificado, passando pelos Estados Intermediários (E), rumo a construção do seu Estado Final (Figura 1).

Figura 1 esquema ilustrativo da Teoria Espacial de Newell e Simon (1972), adaptado de Sternberg (2000).

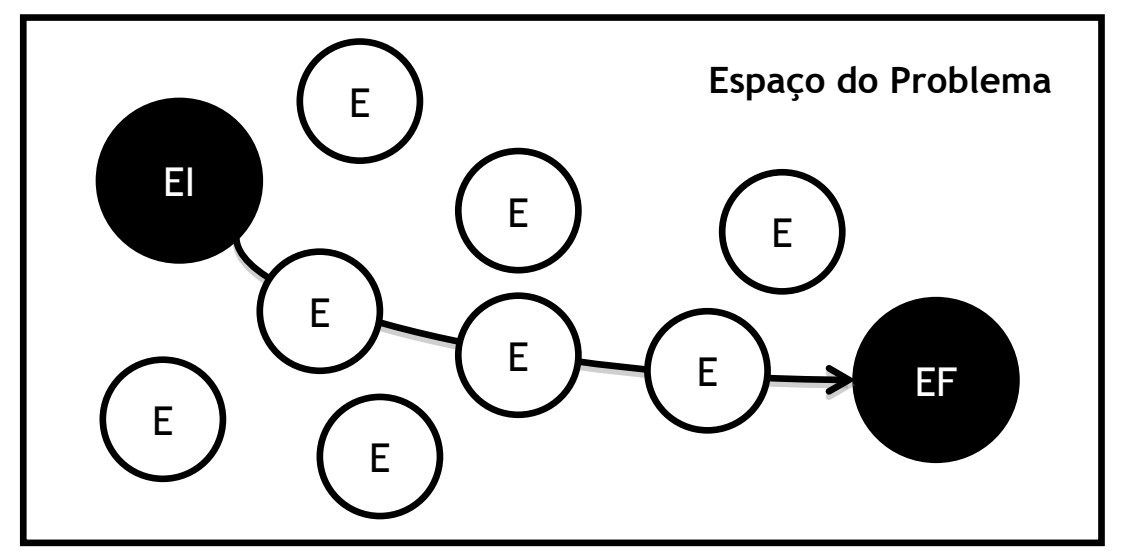


Por essa razão, durante o processo de resolução de um problema, o designer busca a mediação entre os conhecimentos técnicos e as experimentações acerca das possíveis soluções idealizadas. Assim, ele navega pelo espaço do problema enquanto gera as alternativas estruturais ou formais do problema de design. Por isso, esse processo mescla (a) a busca pelo conhecimento técnico, adotando-se os mais diversos tipos de pesquisas, e (b) experimentação das possíveis soluções de acordo com a natureza do problema, relacionando os fragmentos analisados em uma abordagem subjetiva. Para tanto, o designer adota estratégias que visam a superação dos obstáculos encontrados na concepção de novos produtos, sistemas ou serviços, derivadas de seu repertório e alimentadas pela sua experiência de mundo, transpondo o conhecimento ou procedimento para uma aplicação.

Segundo Sternberg (2000), a transposição de um conhecimento ou habilidade de uma situação problemática para outra é denominado Transferência. A Transferência é Positiva quando a resolução de um problema anterior favorece a resolução de um novo problema que guarda analogia por meio da aplicação de uma estratégia ou solução bem sucedida, como o processo de benchmarking, por exemplo. Em contrapartida, quando a experiência na resolução de um problema anterior dificulta ou impede a resolução de outro posterior, tem-se a Transferência Negativa, situação frequente em processos de design em que as soluções projetuais existentes influenciam as decisões do designer de maneira não satisfatória, influenciando a sua abordagem na resolução do problema de design, transformando suas estratégias.

Visando o favorecimento de Transferências Positivas, o processo pode ser propiciado por um gatilho que acione o atalho mental, desde que sejam mantidas as pistas favoráveis à sua recuperação e úteis durante a etapa de projeto. Essas pistas podem auxiliar os designers a elaborarem estratégias operatórias (Abrahão et al., 2009; Guérin et al., 2001), de natureza algorítmica ou heurística, como forma de enriquecer sua representação e favorecer a sua decisão no contexto do projeto (Sternberg, 2000; Anderson 2004; Matlin, 2004).

\section{Heurísticas comuns no processo de design}

Um processo evolutivo natural é a utilização de heurísticas, atalhos mentais, que tornam mais leve e econômico esse processamento cognitivo (Matlin, 2004; Sternberg, 2000; Zsambok \& Klein, 1997; Tversky \& fox, 1995). O raciocínio heurístico é determinado pela natureza do problema e a sua relação com as experiências anteriores das pessoas e suas características individuais. Também é influenciado pelas pressões temporais e está condicionado ao risco envolvido na tarefa, elevando a probabilidade de erro no diagnóstico e na tomada de decisão (Abrahão et al., 2009; Silvino, 2004; Marmaras \& Kontogiannis, 2001; Marmaras \& Pavard, 2000). O processo decisório pode envolver diferentes heurísticas. São apresentadas as seguintes heurísticas 
como frequentes em processos de resolução de problemas: (a) representatividade, (b) disponibilidade, (c) meios-fins, (d) ancoragem e ajuste, (e) gerar e testar e (f) enquadramento.

A heurística de representatividade é definida pela possibilidade de um julgamento prévio da probabilidade de ocorrência de um evento (Matlin, 2004; Sternberg, 2000). Os cálculos probabilísticos sobre a ocorrência do evento são subjetivos, podendo levar também a um julgamento errôneo e resultar em uma ação mal sucedida. Ela ocorre como um atalho que julga a probabilidade de uma associação com base na experiência anterior, mesmo que não haja uma explicação direta para tal associação, como por exemplo, o estereótipo. Essa característica de funcionamento cognitivo humano por atalhos é muito importante para o contexto de resolução de problemas. Aplicado ao design, ele pode favorecer a adoção de técnicas que utilizem esse atalho para favorecer as associações semânticas do designer, ou mesmo permitir que ele evite determinados julgamentos prévios sem a análise mais detalhada do problema em questão. Ainda, esse efeito permite ao designer recuperar traços incompletos de informações pertinentes acerca da estruturação do problema e aplicá-los durante a fase de ideação, guiando a sua experimentação.

A heurística de disponibilidade está associada ao quão facilmente recupera-se uma informação na memória com base em ativações recentes do padrão de ativação (Matlin, 2004; Sternberg, 2000). Por isso, ao se estudar o contexto do problema de design ativamente, essa heurística permite a evocação recorrente das características mais importantes das pesquisas que possam ser relacionadas às alternativas geradas, tornando-as mais pertinentes. O processo contrário também pode ocorrer, no qual uma solução parcial para o problema de design permanece incompleta e a apropriação de um conhecimento técnico específico é relacionado à solução em questão, ligando as duas informações durante o processo de refinamento das alternativas.

A heurística meios-fins ocorre quando o problema é analisado considerando-se o seu estado final, no qual o sujeito age tentando o diminuir a distância entre o estado atual do problema e os seus objetivos finais, dividindo o problema em subcomponentes para viabilizar a sua resolução (Matlin, 2004; Sternberg, 2000). Esse procedimento é muito comum à fase de geração de alternativas no processo de design, que se inicia de maneira fragmentada e passa a sintetizar as soluções intermediárias ao longo do processo.

A heurística da ancoragem e ajuste ocorre de maneira semelhante. Contudo, nesse caso são definidas âncoras intermediárias para o alcance dos objetivos, sendo que são realizados ajustes a posteriori com base em informações extras para aproximar a âncora à solução (Matlin, 2004). Esse processo é também frequente em etapas de projeto, presente na maneira como os desenhos, fluxos, esquemas, listas, modelos são selecionados, visando um refinamento e adequação das ideias até uma alternativa adequada para se resolver o problema. 
De maneira semelhante, a heurística de gerar e testar, comumente chamada de tentativa e erro, visa o deslocamento rumo ao estado final do problema por aproximação. Contudo, ela ocorre de maneira não sistemática, quando o curso da ação é gerado apenas como uma tentativa de transformação do estado atual e o resultado é avaliado posteriormente (Sternberg, 2000). Esse é um procedimento de avaliação, no qual se tenta modificar o estado atual do problema, verificando-se apenas posteriormente a pertinência do novo estado obtido. Geralmente, essa é uma estratégia que auxilia o designer a reformular a sua representação sobre o problema de design, evitando problemas de fixação em um estado específico, descritos detalhadamente mais adiante.

Ainda sobre a representação construída do problema, existe o efeito de enquadramento, que demonstra que os resultados de uma decisão geralmente são influenciados por dois fatores: o contexto no qual a escolha foi realizada e a maneira como o problema foi formulado (Matlin, 2004). Esse é um fator relevante para o processo de design, no qual a abordagem de voltada para o problema visa uma reestruturação do problema antes da sua resolução. Uma representação inadequada do problema pode dificultar o processo de ideação do designer ou mesmo levar a uma solução inadequada para o contexto. Os principais efeitos relacionados à Representação Inicial do problema são discutidos mais adiante.

\section{Processos de análise e síntese (Modelo Genérico de Design)}

Essas heurísticas são atalhos que contribuem para a construção de estratégias de resolução de problemas, alimentadas por uma experiência anterior, vinculando a nova experiência ao conhecimento já adquirido. Elas são fatores essenciais no processo de subjetivação que envolve a relação dos conhecimentos técnicos e experimentações no processo de design. Além disso, elas ilustram que a atividade de resolução do problema pode ocorrer de forma concomitante ao domínio dos elementos componentes do problema, não sendo necessária uma divisão rígida dessas etapas. Contudo, Newel e Simon (1972, em Sterberg, 2000) apontam que essa apropriação acerca dos componentes do problema, denominada Estruturação do Problema, é fundamental e, em muitos casos, chega a ser determinante do seu sucesso efetivo.

No mesmo sentido, Saffer (2006) aponta a presença frequente de dois ciclos de expansão e retração em processos de design. O primeiro ciclo consiste na realização de pesquisas que visam fragmentar o objeto de estudo em uma análise do contexto dos produtos ou serviços designados, que depois são agrupadas em uma síntese de ideias estruturadas sobre as suas principais características pretendidas, os seus requisitos. O segundo ciclo se inicia em um processo abrangente de idealização, que vai sendo refinado quando comparado aos 
princípios oriundos do ciclo anterior, até a proposta de uma solução completa (Vianna et al., 2012). Ao se relacionar com o modelo de Newel e Simon, esses ciclos se referem às etapas de Estruturação e Resolução do Problema, respectivamente. Esse processo é ilustrado pela Figura 2.

Figura 2 Processo de design baseado no processo de Resolução de Problemas.

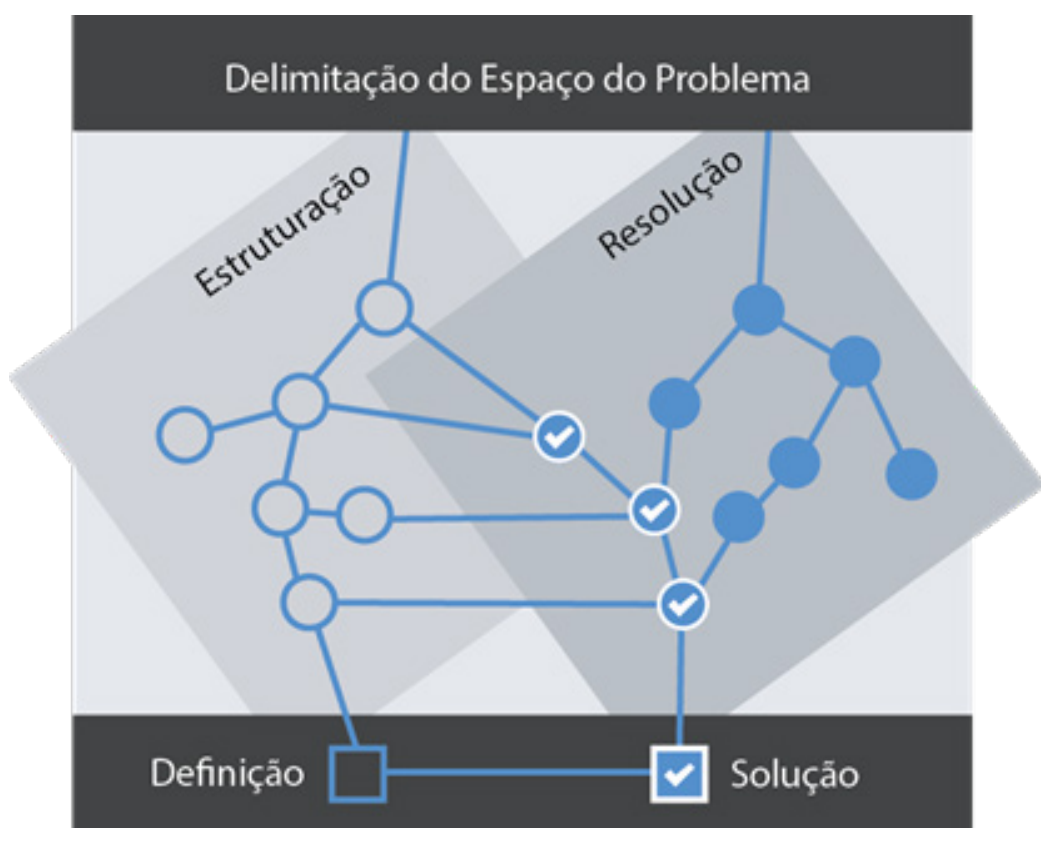

Esse processo de duplos ciclos aumenta a probabilidade de sucesso da alternativa sugerida pelo designer, e por isso é a base de seu método (Saffer, 2006). Cabe ressaltar que, conforme explicitado anteriormente, o processo de resolução do problema não precisa ser posterior à sua estruturação, de forma que ambos os ciclos podem ocorrer concomitantemente. Esse processo é ilustrado pela Figura 3, que demonstra um Modelo Genérico de Design em que as etapas não seguem uma estrutura linear, e as características pretendidas para os produtos, sistemas ou serviços surgem de pesquisas acerca do seu contexto ou de experimentações de suas formas ou estruturas possíveis. 
Figura 3 Modelo Genérico de Design baseado no processo de Resolução de Problemas.

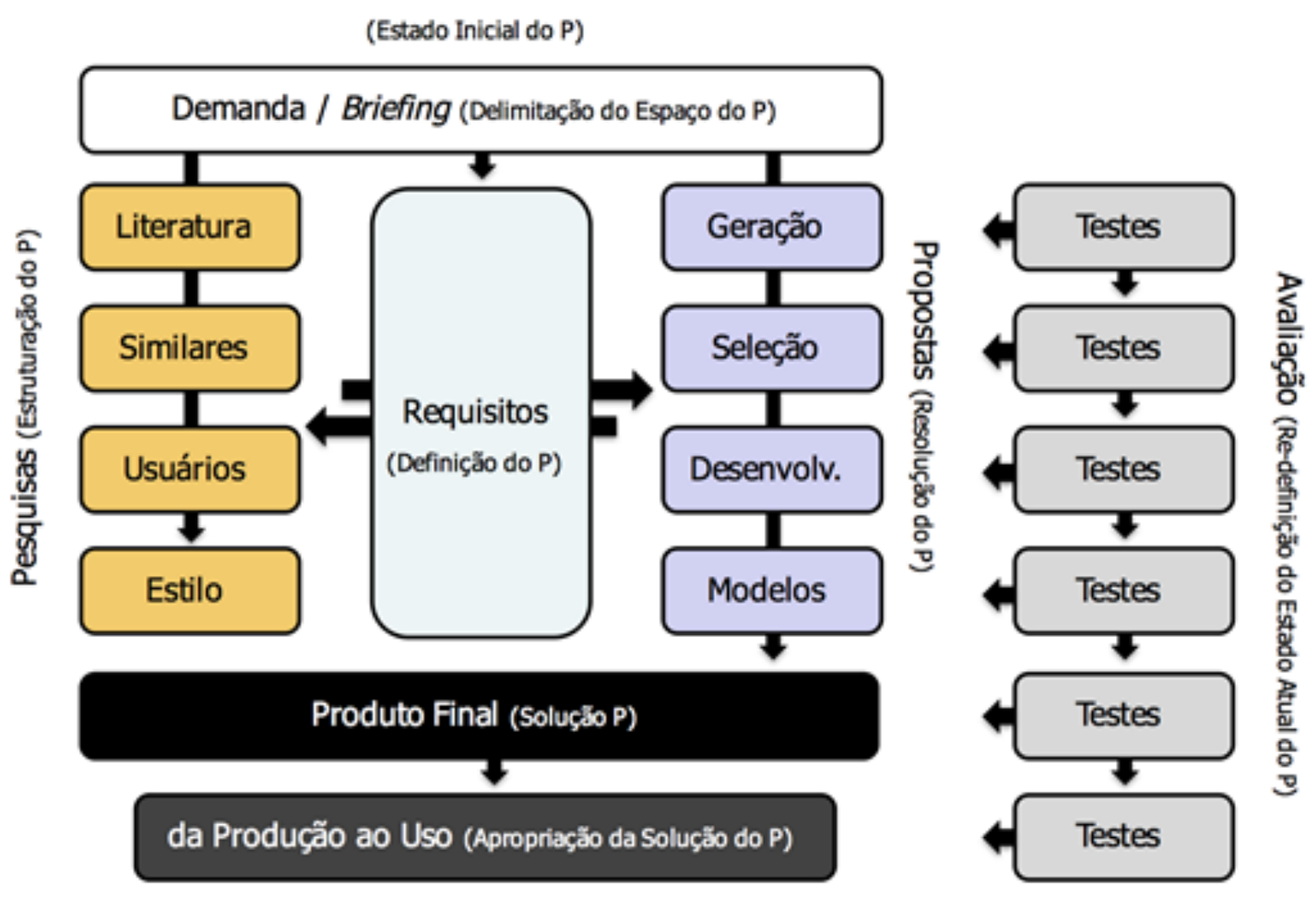

(Estado Final do P)

De acordo com o modelo sugerido, o designer inicia o projeto na (a) Delimitação do Problema, que consiste no entendimento sobre o Espaço do Problema, definindo-se os seus objetivos, motivações, constrangimentos, e todas as informações necessárias sobre quem demanda o problema. Nessa etapa, geralmente são realizadas entrevistas com responsáveis, o briefing, são discutidas a Proposta e o Contrato de Trabalho. Em seguida, o designer pode optar por compreender melhor o problema de design em questão, as características pretendidas para a solução ou tentar solucioná-lo diretamente.

O primeiro ciclo de análise e síntese consiste na (b) Estruturação do Problema, no qual as pesquisas são realizadas para ampliar a confiabilidade da solução final proposta. Nesse momento, diversas técnicas de pesquisa podem ser adotadas, como a análise documental, questionários, entrevistas, grupos focais, observações globais ou sistemáticas (Cozby, 2003; Marconi \& Lakatos, 2008; Martins \& Theóphilo, 2009), em diferentes tipos de delineamento, ou mesmo contextos de pesquisas etnográficas (Nardi, 1997) ou de avaliações ergonômicas (Abrahão et al., 2009), entre outras possibilidades. Lembra-se que a lógica da construção do conhecimento não segue um campo epistemológico específico, considerando-se que o objetivo do projeto não é a produção do conhecimento sobre os fenômenos naturais ou sobre o comportamento das pessoas, mas um conhecimento técnico específico acerca do problema de design (Bonsiepe, 1997). Geralmente, quando o designer realiza uma pesquisa 
empírica nessa etapa de projeto, o seu objetivo é obter uma nova representação acerca da solução, do seu contexto ou de seus usuários, mudando o seu ponto de vista sobre as relações entre a sua forma/ estrutura e as suas funções/relações de significado. Para isso, pode utilizar técnicas mais específicas, como observação fly on the wall, observações globais participativas, uso de card sortings, avaliações de consensos e divergências com grupos focais, entre outras técnicas (Saffer, 2006; Portigal, 2013). O modelo genérico ilustra a etapa de Estruturação com tópicos comuns em pesquisas de design, como a busca na literatura por normas e legislações específicas, conceitos relevantes para o projeto; o estudo de produtos concorrentes ou similares; o estudo das características ou comportamentos de usuários atuais ou potenciais; estudos de estilo, identidade e marca.

O outro momento de análise e síntese do processo de design possui natureza distinta, no qual o designer tenta buscar soluções de configurações formais ou estruturais na fase de (c) Resolução do Problema. Nessa etapa a experimentação é o guia condutor do processo de ideação e geração de soluções alternativas de design. Diversas técnicas podem ser utilizadas, como os brainstormings, o Perfil de Personalidade do Produto, análise da Arquitetura do Produto por desenhos técnicos, protótipos de baixa fidelidade, blueprints e wireframes, desenhos livres, entre outras (Pazmino, 2013; Saffer, 2006; Baxter, 1998). Essa etapa é ilustrada no modelo por processos de geração, seleção e desenvolvimento de alternativas para o produto, sistema ou serviço. Destaca-se que a maneira de representar o problema e as suas soluções provisórias podem ser determinantes como instrumentos de auxílio a sua resolução, sendo comumente empregados símbolos, matrizes, diagramas, imagens, fluxos, entre outros (Saffer, 2006; Matlin, 2004).

Na mediação entre esses dois ciclos, o designer realiza a (d) Definição do Problema, visando compreendê-lo melhor a partir dos estudos e experimentações realizadas. Essa etapa consiste em redefinir o problema de design da melhor maneira possível, detalhando os seus atributos, que foram embasados pela Estruturação e devem ser tangibilizados pela Resolução do Problema. Diversas técnicas focam nessa etapa do projeto, como o modelo Kano (Baxter, 1998), o Perfil Semântico do Produto (Jordan, 2000), o Quality Function Deployment - QFD (Desdobramento da Função Qualidade), as listas de requisitos, entre outras possibilidades. É importante frisar que essas etapas podem ser navegadas de maneira não linear a depender da natureza do projeto, permitindo que os designers estruturem o problema enquanto o resolvem de maneira dinâmica e flexível, conforme apontado anteriormente na teoria de Newell e Simon (1972).

Ao final desses ciclos entre as etapas apresentadas, o designer seleciona uma (e) Solução para o Problema, apresentando os renderings, desenhos técnicos, arquivos fechados para impressão, detalhamentos técnicos de produção, relatórios, apresentações, modelos, entre outros. Além disso, durante todo o projeto, os testes 
podem ser realizados com produtos similares existentes, protótipos de baixa fidelidade, funcionais ou modelos do sistema projetado, visando avaliar as dimensões de segurança, funcionalidade, acessibilidade, encontrabilidade (Morville, 2005), usabilidade ou agradabilidade (Jordan, 2000; Khalid \& Helander, 2006) da interface concebida para o produto, sistema ou serviço. Por isso, os testes podem ser considerados como avaliações das soluções formais e estruturais do problema de design a partir dos parâmetros definidos pelo seu processo de estruturação.

A sequência de ações dentro do Modelo Genérico de Design não segue uma ordem rígida, e o processo evolui de maneira mais rápida quando as etapas são alternadas de forma ágil e repetidas frequentemente, mas não sempre na mesma ordem (Moggridge, 2007), ilustradas pelo esquema apresentado na Figura 4. É importante lembrar que, geralmente, o processo é conduzido por uma equipe, e não por uma única pessoa. Por isso, os diferentes papéis assumidos pelos profissionais envolvidos podem ser personificados por um ou mais integrantes da equipe. Ainda, em composições de equipes com formação heterogênea, modelos comuns de representação das informações pertinentes ao projeto se mostram de suma importância.

Por exemplo, durante o projeto de jogos eletrônicos, os desenvolvedores, artistas, músicos e game designers devem ser capazes de compreender as informações com naturezas distintas, mas igualmente vinculadas ao projeto, de uma maneira coerente, sintetizando todas as propriedades esperadas no sistema desenvolvido de forma coesa.

Figura 4 Elementos componentes do processo de design, retirado de Moggridge (2007).

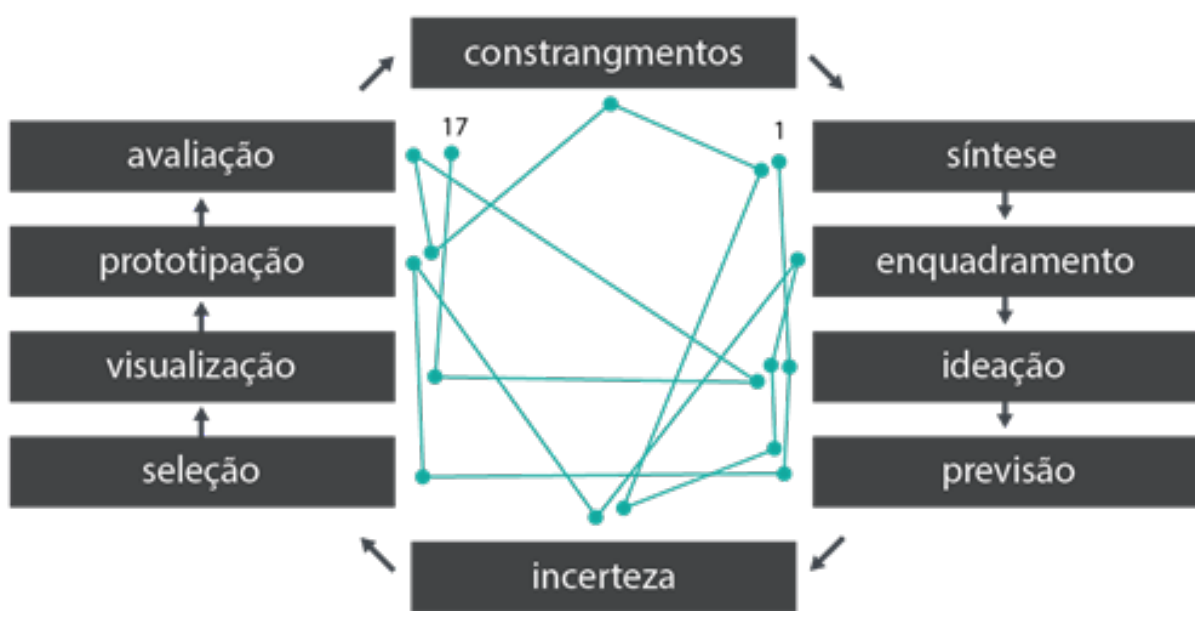

No esquema proposto por Moggridge (2007), o processo de design é composto por dez elementos que definem a natureza da ação do designer ao longo do projeto: restrição, síntese, enquadramento, ideação, previsão, incerteza, seleção, visualização, prototipação e avaliação (tradução livre do autor). As linhas verdes representam um 
percurso realista e não linear da ordem de navegação entre as etapas e a linha preta ilustra seu caráter cíclico (iterativo). De acordo com o autor, os processos mais produtivos geralmente estão relacionados à navegação livre pelos elementos componentes no processo de design.

\section{0 processo de arquitetura cognitiva}

A adoção dessa lógica no processo de design permite não só o entendimento do papel do designer em cada uma das diferentes etapas de projeto, compreendendo o contexto do produto, sistema ou serviço projetado ou experimentando as suas possíveis soluções, mas permite também a adoção de algumas das contribuições da Gestalt para o contexto de resolução de problemas. Para descrevê-las, é necessário primeiramente entender o modelo de arquitetura cognitiva proposto por Anderson (1983), o Cognitive Arquitecture Process - CAP*.

Esse modelo pressupõe a existência de redes semânticas que agregam os conhecimentos declarativos e procedimentais em uma estrutura que se assemelha à Memória de Longo Prazo (MLP) dos modelos clássicos de memória. Essas redes de conceitos são conectadas com base em relações de significado e são organizadas com base na sua frequência de utilização, favorecendo ou inibindo determinadas associações por frequência de co-ativação. O efeito ocorre porque por essa rede transita um padrão de ativação, uma energia com quantidade determinada que se espalha pela rede, responsável pela ativação de seus nós, que equivale à Memória de Trabalho (MT), antiga Memória de Curto Prazo dos modelos clássicos. A transmissão dessa energia reforça os laços entre os nós, inibindo os caminhos alternativos de dispersão. A partir do uso dessa estrutura, os estímulos externos e internos, mediados por processos perceptivos e atencionais, resultam em padrões altamente dinâmicos, que retratam reconstruções simbólicas incompletas dos objetos originais. Um esquema ilustrativo de funcionamento do $\mathrm{CAP}^{*}$ pode ser visto na Figura 5 .

Figura 5 Esquema ilustrativo do Cognitive Arquitecture Process - CAP*, adaptado de Sternberg (2000).

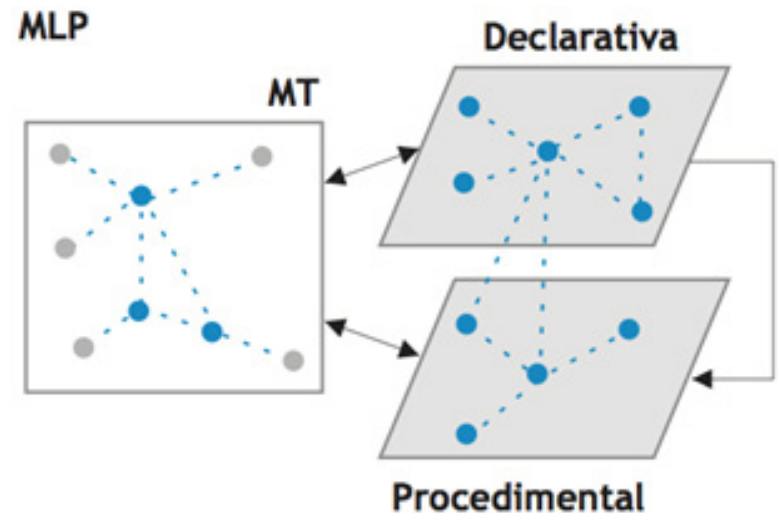


Essas características permitem ao modelo explicar as situações em que padrões de ativação específicos favorecem a evocação de determinado conteúdo semântico ou operacional em detrimento de outros. Por isso, a depender da situação específica do contexto, a recuperação de uma informação pode se tornar um desafio, mesmo quando as pessoas tentam realizar essa tarefa voluntariamente. Ao se mudar a situação, a energia do padrão de ativação segue um outro caminho na rede, e a informação pode ser acessada. Essa lógica é fruto de um processo evolutivo que surge pela economia de recursos em sua utilização, e permite o entendimento da adoção das estratégias heurísticas descritas como atalhos mentais em contextos de resolução de problemas.

O processo de distribuição dessa energia na rede semântica também sofre influência de distintos processos afetivos, em situações de estados de afeto positivo ou negativo, favorecendo a ativação de emoções ou sentimentos presentes nas experiências das pessoas, mescladas com a dimensão cognitiva e ativando representações na Memória de Trabalho (Ledoux, 2001; Aboulafia \& Bannon, 2004; Khalid, 2006). Evolutivamente, os componentes cognitivos e afetivos foram associados e aprimorados para gerar uma atribuição de significado e valor, respectivamente (Norman, 2004).

Além disso, o modelo também permite explicar algumas características de funcionamento da memória que podem auxiliar o designer ao longo do projeto em um processo de metacognição. Essas estratégias são adotadas em diferentes ferramentas de design, voltadas para o estímulo da criatividade durante a fase de ideação do projeto. Nesse contexto, a criatividade é entendida como a possibilidade de se realizar associações não convencionais desses campos semânticos, dados os padrões já estabelecidos de Representações Sociais existentes nos grupos.

Um exemplo do uso desse atributo pode ser identificado na técnica conhecida como brainstorming. Ela consiste na exposição de ideias e associações sem julgamentos para solucionar um determinado problema. O objetivo é permitir a quebra das representações comuns dos integrantes dos grupos por meio das ideias apresentadas. Por isso, o fato de não se avaliar e tolher as ideias dos participantes durante a fase de proposições é tão importante. Os seus padrões de ativação mudam dinamicamente de acordo com as soluções intermediárias, que permitem o acesso a um outro caminho na rede de conceitos, aumentando a probabilidade de se obter uma associação não convencional que seja relevante.

\section{Metacognição e criatividade}

Na maior parte dos casos, o objetivo das técnicas que visam criatividade é o de permitir que o designer modifique o seu padrão de ativação ao resolver o problema. Isso permite que ele tenha uma nova representação do Estado Inicial do problema, reformulando a maneira 
de compreendê-lo. Nesses casos, vieses causados por obstáculos de representação inicial, também conhecida como configuração mental (Matlin, 2004), podem ser superados mais rapidamente. Por esse motivo, muitas vezes os designers são estimulados a formular o problema da forma mais ampla o possível, visando não restringir as possibilidades de solução logo na sua primeira etapa. Além disso, essa flexibilidade permite ao designer recombinar as informações obtidas na fase de estruturação do problema de maneiras não convencionais, aumentando o seu leque de opções e repertório.

Somam-se aos Problemas de Representação Inicial outras situações semelhantes que causam o efeito conhecido como Persistência do Set (Sternberg, 2000). Nessas situações, o padrão de representação é evocado sistematicamente ao se tentar resolver determinado problema, mas os caminhos na busca de uma associação pertinente não são favorecidos. Nesses casos, os problemas de representação inicial se tornam persistentes, dificultado a adoção de novas estratégias. Diversas técnicas de experimentação em design, como a Sinética, a Analogia (Matlin, 2004), ou o MESCRAI (Baxter, 1998), são utilizadas para se evitar dificuldades dessa natureza durante a fase de ideação do projeto.

Uma outra situação semelhante é conhecida como Fixidez Funcional (Matlin, 2004; Sternberg, 2000). Nessas situações, o Problema de Representação Inicial persiste devido a uma forte associação à função já conhecida de um determinado objeto ou sistema, dificultando a possibilidade de visualizações de outras abordagens ou estímulos mais pertinentes para a resolução do problema. Nesse sentido, técnicas de Benchmarking são estimuladas para que soluções pertinentes adotadas por produtos concorrentes, ou mesmo produtos de nichos completamente distintos, mas que possuem alguma similaridade na dimensão avaliada, possam ser transpostas para o projeto em andamento.

O modelo também explica o processo de Insight (Matlin, 2004; Sternberg, 2000), no qual um problema é resolvido somente depois de um período de afastamento da intenção de resolvê-lo. Esse efeito ocorre porque a representação se modifica drasticamente ao se realizar outras atividades de diferentes naturezas, mas o cérebro é capaz de manter ativa a lembrança de que a solução para o problema ainda não foi encontrada. Os problemas que se beneficiam dessa abordagem geralmente demandam uma imersão em conhecimentos relacionados e abordagens indutivas na busca pela solução (Matlin, 2004), como os problemas de design.

A adoção dessa estratégia de maneira explícita no processo de design foi proposta na escola de Ulm, denominada de Processo Criativo Quântico (Wollner, 2010). O processo é descrito como uma sequência de passos para o designer durante a fase de projeto, que consistem em: (a) preparação, (b) incubação, (c) insight criativo ou comunicação Gestalt, (d) manifestação e (e) comunicação. O modelo descreve o uso do processo de Insight como o centro do método de 
design, no qual o designer deve primeiramente estudar o problema de design, depois realizar outras atividades que propiciem a associação criativa, realizar a associação, voltar a vincular a ideia explicitamente ao projeto, realizando todos os ajustes técnicos necessários e documentá-la, respectivamente.

É importante observar ainda que, durante a execução do projeto, muitas vezes etapas mais específicas do processo de design se caracterizam como problemas de não-insight. Nesses casos, geralmente as soluções são beneficiadas por uma abordagem descendente, partindo-se, por exemplo, de uma estratégia algorítmica para se obter a solução (Matlin, 2004).

\section{Sentidos de investigação}

A compreensão de design enquanto Processo de Resolução de Problemas permite o entendimento do campo epistemológico do design como projeto, que relaciona conhecimentos oriundos de diferentes abordagens científicas, artísticas e de tecnologias disponíveis com intenção de transformação e inovação sociocultural por meio da articulação da interface entre os usuários e os artefatos projetados (Bonsiepe, 1997). Para tanto, o método específico de cada projeto depende da natureza do problema abordado, devido a sua necessidade de se relacionar intimamente ao seu contexto, e também devido ao caráter interdisciplinar intrínseco ao processo de design (Cipiniuk \& Portinari, 2006).

A depender do contexto do produto, sistema ou serviço projetado, do seu nível de complexidade, dimensão ou das relações com seus interatores, essa abordagem metodológica pode seguir um processo de orientação ascendente (bottom-up), na qual se tem um projeto por desvelamento em vez de determinístico. Nesses casos, as decisões são tomadas a partir da experimentação, adequando o planejamento ao contexto de complexidade inerente ao projeto, visando-se fragmentar os seus diferentes segmentos em núcleos inteligíveis e gerenciáveis de projeto, além de adequar as soluções propostas ao contexto em processos de balanceamento e ajustes cíclicos e contínuos (Moraes, 2010; Vassão, 2008; 2010; Vianna et al., 2012).

Nesse sentido, entende-se que o processo de design em contextos de sistemas complexos e dinâmicos deve ser adaptativo, com intuito de se manter pertinente ao longo do tempo. São exemplos dessas abordagens os procedimentos em ciclos discutidos nas técnicas de lean $U X$, voltados para uma rápida prototipação e avaliação, com foco na adaptabilidade do projeto (Cothelf \& Seiden, 2013). Ainda, são muito frequentes em abordagens de Design Thinking, na qual emprega-se a abordagem de design para a resolução de problemas de outras naturezas (Vianna et al., 2012), dividindo o método em ciclos que alternam entre as etapas de Imersão, Ideação, Prototipação e Avaliação continuamente até que se obtenha a solução final do problema. 


\section{0 contexto do Design da Informação}

Apesar de sua origem vinculada às áreas de design gráfico e editorial (Shedrof, 2000), o campo atual do Design da Informação se constitui cada vez mais dinâmico e complexo devido às potencialidades oriundas do avanço tecnológico. Interfaces portáteis, vestíveis, lúdicas ou ubíquas compõem uma ecologia de interações cada vez mais dispersas, automatizadas e com comportamentos inteligentes e contextualizados. Assim, a cada dia se torna mais pertinente a conversão de fluxos de dados não inteligíveis em informação com significado para os usuários desses sistemas para que sejam capazes de se apropriar efetivamente dos conteúdos e funções disponibilizadas. Contudo, o processo de concepção das estruturas e interfaces desses sistemas se torna um desafio frente ao volume de informações necessárias aos designers para uma compreensão adequada do problema. Ainda, a questão da adaptabilidade dos projetos se intensifica, demandando agilidade e otimização dos processos de design.

Nesse sentido, a adoção de estratégias de metacognição na condução do projeto se torna pertinente. Por meio da aplicação intencional de estratégias de estruturação e resolução do problema de design, é possível favorecer a compreensão acerca do contexto do projeto, assim como navegar de maneira mais fluida pela etapa de ideação, fragmentando e sintetizando os conhecimentos e proposições rumo à solução do problema.

Ainda, considerando-se o campo do Design da Informação, que visa disponibilizar informações, de maneira clara e objetiva para os seus usuários (Quintão \& Triska, 2013; Mineiro 2011; Portugal, 2010), a compreensão acerca do processo de resolução do problema de navegação também se faz pertinente. Em contextos de sistemas interativos, questões relativas à facilidade de uso, tempo de aprendizado e erros de navegação estão relacionados ao nível de compreensão que o usuário tem acerca da sua interface. Assim, em concomitância com os instrumentos de mensuração de usabilidade, como as técnicas pós-evento como o Post-Study System Usability Questionnaire (PSSUQ), os parâmetros intrínsecos de usabilidade (Nielsen, 1990; Shneiderman, 1986) ou as avaliações dos comportamentos de navegação (Abrahão et al., 2009), a compreensão do processo de resolução do problema de navegação dos usuários pode beneficiar as soluções apresentadas, assim como o seu processo de avaliação, permitindo inferências dos designers acerca dos processamentos cognitivos dos usuários que determinaram suas estratégias de ação (Marmaras \& Pavard, 2000). Com isso, o diagnóstico da situação pode ser beneficiado, levando à construção de soluções pertinentes que forneçam as pistas necessárias para o sucesso da navegação. 


\section{Considerações}

Portanto, a partir da abordagem metodológica e fundamentação teórica acerca do processo de design descrito, espera-se permitir que o designer seja capaz de ter mais controle e propriedade acerca do seu papel. Nesse sentido, ele pode adotar estratégias para lidar com as diferentes situações do problema a ser resolvido, compreendendo as diferentes naturezas das etapas de estruturação e resolução do problema, assim como adotar técnicas que permitam otimizar etapas de proposição e avaliação das soluções desenvolvidas. Ainda, o repertório apresentado pode permitir a realização de inferências acerca das estratégias dos usuários durante o seu comportamento de navegação.

No campo do Design da Informação, espera-se que os processos de metacognição apoiem os designers na concepção de interfaces informacionais enquanto sistemas complexos e dinâmicos, favorecendo a gestão de um número elevado de variáveis contextuais durante a estruturação do problema e permitindo uma avaliação mais efetiva das soluções concebidas propiciando o aspecto de adaptabilidade do projeto.

Apoiado na natureza mediadora do processo de design, o que se espera é sugerir que os designers percorram caminhos tão diversos e, ao mesmo tempo, comuns, unindo a Arte e a Ciência pela sua principal característica: o seu espírito inventivo (Santaella, 2012). Dessa maneira, processos de pensamento divergentes, comuns ao design e à arte; e convergentes, presentes em abordagens científicas, são mesclados na busca por produção de conhecimento, experimentação e projeto de soluções inovadoras em etapas concretas e abstratas dos projetos (Maeda, 2010; 2013; Cross, 2011; Brown, 2009).

\section{Referências}

ABOULAFIA, A.; BANNON, L. J. Understanding affect in design: an outline conceptual framework. Theoretical Issues in Ergonomics Science, 5(1), 4-15. 2004.

ABRAHÃO, J. I.; SZNELWAR, L.; SILVINO, A. M. D.; SARMET, M. M. e PINHO, D. Introdução à ergonomia: da prática à teoria. São Paulo: Edgard Blücher LTDA, 2009.

ANDERSON, J. R. Psicologia Cognitiva e Suas Implicações Experimentais. Rio de Janeiro: LTC, 2004.

BAXTER, M. Projeto de produto. São Paulo: Edgard Blücher,1998.

BONSIEPE, G. Design: do material ao digital. Florianópolis: IEL/Fiesc, 1997. . Metodologia Experimental: Desenho Industrial. Brasília: CNPq - Coordenação Editorial, 1984.

BROWN, T. Change by Design. HarperCollins, 2009. 
CIPINIUK, A.; PORTINARI, D. B. Sobre Métodos de Design. In: COELHO, L. A. L. (org). Design Método. Rio de Janeiro, Teresópolis: PUC-Rio; Novas Idéias, 2006.

COZBY, P. C. Métodos de Pesquisa em Ciências do Comportamento. São Paulo: Editora Atlas, 2003.

CROSS, N. Design Thinking: Understanding How Designers Think and Work. New York: Berg, 2011.

GOTHELF J.; SEIDEN J. Lean UX: Applying Lean Principles to Improve User Experience. O'Reilly: 2013.

GUÉRIN, F.; LAVILLE, A.; DANIELLOU, F.; DURAFFOURG, J. e KERGUELEN, A. Compreender o trabalho para transformá-lo: A prática da Ergonomia. Tradução de: L. Sznelwar et al. São Paulo: Edgard Blücher LTDA, 2001.

JORDAN, P. W. Designing Pleasurable Products. London: Taylor \& Francis, 2000.

KHALID, H. M. Embracing diversity in user needs for affective design. Applied Ergonomics, 37. 409-418. 2006.

KHALID, H. M. e HELANDER, M. G., Customer Emotional Needs in Product Design. Concurrent Engineering, v. 14, n.3, set. 2006, p. 197-206.

LEDOUX, J. O Cérebro Emocional: Os misteriosos alicerces da vida emocional. Rio de Janeiro: Objetiva, 2001.

LÖBACH, B. Design industrial. São Paulo: Edgard Blücher, 2001.

MAEDA, J. Artists and Scientists: More Alike Than Different. Scientific American Blog Network, Guest Blog: 2013. Recuperado em agosto de 2013: http://blogs.scientificamerican.com/guest-blog/2013/07/11/ artists-and-scientists-more-alike-than-different/ Innovation is born when art meets science. Em Lamont, T. (entrevista), The Observer. The Guardian: Sunday 14 November 2010. Recuperado em dezembro de 2012: http://www.guardian.co.uk/technology/2010/nov/14/ my-bright-idea-john-maeda

MALDONADO, T. Design Industrial. Edições 70, Lisboa, 1999.

MARMARAS, N. \& KONTOGIANNIS, T. Cognitive Task. Em: G. Salvendy, Handbook of Industrial Engineering. New York: John Wiley \& Sons, 2001.

MARMARAS, N. e PAVARD, B. Problem-Driven Approach to the Design of Information Technology Systems Supporting Complex Cognitive Tasks. Cognition, Technology \& Work. London: Springer-Verlag London Limited, 2000.

MATLIN, M. W. Psicologia Cognitiva. Rio de Janeiro: LTC, 2004.

MARCONI, M. A.; LAKATOS, E. M. Metodologia Científica - 5 $5^{\text {a }}$ Edição - revisada e ampliada. São Paulo: Editora Atlas, 2008.

MARTINS, G. A.; THEÓPHILO, C. R. Metodologia da Investigação Científica para Ciências Sociais Aplicadas - 2 a Edição. São Paulo: Atlas, 2009.

MINEIRO, Érico F. Design da informação, modelos mentais e a gestão da inovação: articulações possíveis. InfoDesign v. 8, n. 3. São Paulo: 2011.

MORAES, D. Metaprojeto: o design do design. São Paulo: Edgard Blücher, 2010.

MOGGRIDGE, B. Designing Interactions. USA: The MIT Press, 2007.

MUNARI, B. Das coisas nascem coisas. São Paulo: Martins Fontes, 1998.

NIELSEN, J.; MOLICH, R. Heuristic evaluation of user interfaces. In: Proceedings ACM CHI 90 Conference. Seattle: 1990. p. 249-256. 
NORMAN, D. Emotional Design: Why do we love (or hate) everyday things. New York: Basic Books, 2004.

PAPANEK, V. Design for the real world: human ecology and social change. Chicago: Academy Chicago Publishers, 2000.

PAZMinO, A. V. Como se cria: 40 métodos para o design de produtos. São Paulo: Blucher, 2013.

PORTIGAL, S. Interviewing Users: How to Uncover Compelling Insights. New York, EUA: Rosenfeld Media, LLC, 2013.

PORTUGAL, C. Questões complexas do design da informação e de interação. InfoDesign v.7, n. 2. São Paulo: 2010.

QUINTÃO, F. S.; TRISKA, R. Design de informação em interfaces digitais: origens, definições e fundamentos. Revista Brasileira de Design da Informação. InfoDesign v. 10, n. 2. São Paulo: 2013.

SAFFER, D. Design for interaction. Peachpit Press, 2006.

SANTAELLA, L. A relevância da arte-ciência na contemporaneidade. Em Venturelli, S. (org.) ; Rocha, C. (org.) ; Medeiros, M. B. de (org.). ART - Arte e Tecnologia: Modus Operandi Universal. 1. ed. Brasília: Programa de pósgraduação em arte da UnB, 2012. 103 a 111.

SANT'ANNA, Hugo Cristo. Design sem Designer. Serra: Edição do autor, 2013.

SHEDROFF, N. Information interaction design: a unified field theory of design. In: JACOBSON, Robert (ed.). Information design. Cambridge (MA): The MIT Press, 2000.

SHNEIDERMAN, Ben. Designing the User Interface: Strategies for Effective HumanComputer Interaction. 1st edition. Addison-Wesley, 1986.

SILVINO, A. M. D. Ergonomia Cognitiva e Exclusão Digital: a Competência como Elemento de (re)Concepção de Interfaces Gráficas. Tese de Doutorado, Universidade de Brasília, Brasília, 2004.

SILVINO, A.M.D.; SILVA, T. B. P. ; SARMET, M. M. Exclusão Digital: Dimensões de análise de sítios web. In: XV Congresso Brasileiro de Ergonomia, 2008, Porto Seguro. Anais do XV Congresso Brasileiro de Ergonomia, 2008.

STERNBERG, R. J. Psicologia Cognitiva. Porto Alegre: ArtMed Editora, 2000.

TVERSKY, A.; FOX, C. R. Weighing Risk and Uncertainty. Psychological Review, Vol. 102, No. 2, pp. 269-283. 1995.

VASSÃO, C. A. Arquitetura Livre: Complexidade, Metadesign e Ciência Nômade. Tese de Doutorado, FAU USP, São Paulo, 2008. . Metadesign: ferramentas, estratégias e ética para a complexidade. São Paulo: Blucher, 2010.

VIANNA, M.; VIANNA, Y.; ADLER, I. K.; LUCENA, B.; RUSSO, B. Design Thinking: Inovação em Negócios. Rio de Janeiro: MJV Press, 2012.

WOLLNER, A. Palestra na 4a Semana de Design na Uiversidade de Brasília - UnB. 2010.

ZSAMBOK, C. E.; KLEIN, G. Naturalistic Decision Making. Lawrence Erlbaum Associates, 1997. 


\section{Sobre o autor}

Tiago Barros Pontes e Silva

PhD, Universidade de Brasília (UnB), Brasil <tiagobarros@unb.br>

Artigo recebido em 18 abr. 2015,

aprovado em 07 dez. 2015. 